Classification

Physics Abstracts

$75.10 \mathrm{H}$

\title{
Random antiphase state and frustration in two dimensions
}

\author{
R. Maynard and R. Rammal \\ Centre de Recherches sur les Très Basses Températures, \\ Centre National de la Recherche Scientifique, BP 166X, 38042 Grenoble Cedex, France
}

(Reçu le 19 février 1982, accepté le 22 mars 1982)

\begin{abstract}
Résumé. - Le diagramme de phase du modèle de frustration - spins d'Ising voisins en interaction aléatoire $\pm J$ - sur un réseau carré est étudié dans le plan $(T, x): T$ température et $x$ concentration d'interactions antiferromagnétiques - $J$. A température nulle, l'ensemble des états fondamentaux peut être obtenu sans approximation par un nouvel algorithme de la théorie des graphes. La carte des liaisons rigides et des spins solidaires est établie pour tous les fondamentaux d'un échantillon donné. A concentration intermédiaire $x$ une nouvelle phase est découverte où les lignes de parois magnétiques prolifèrent spontanément. $\mathrm{A} T \neq 0 \mathrm{~K}$, la méthode simpl(iste) du groupe de renormalisation dans l'espace réel confirme l'existence de cet état d'antiphase aléatoire.
\end{abstract}

\begin{abstract}
The phase diagram of the frustration model of random interactions $\pm J$ between nearest neighbour Ising spins on a square lattice is studied in the $(T, x)$ plane : $T$ temperature, $x$ concentration of antiferromagnetic interactions. At $T=0 \mathrm{~K}$ exact ground states are generated by a new algorithm of graph theory and maps of rigid bonds or solidary spins for all ground states are obtained. At intermediate $x$ it is found a new phase made up of erratic magnetic walls (lines). At $T \neq 0 \mathrm{~K}$ a simple renormalization group approach confirms the existence of the random antiphase state.
\end{abstract}

It is currently admitted that, in two dimensions, the standard model of Ising spin glasses based on a symmetrical distribution of random interaction $J, p(-J)=p(J)$, does not exhibit any spin glass phase but only paramagnetism at $T \neq 0 \mathrm{~K}$. By varying the concentration $x$ of negative interactions (from $x=0.5$ to $x=0$ ), a phase diagram $(x, T)$ is explored and the previous attempts [1] concluded to the existence of a transition from paramagnetism to ferromagnetism at low concentration $x$ and nothing else. Using a new algorithm for generating exact ground states we have found a new phase nested near the ferromagnetism region that we have called « random antiphase state » (RAS).

1. Frustration and the Chinese postman's problem - The Hamiltonian which has been studied here is written :

$$
\mathscr{H}=-\sum_{(\mathbf{i}, \mathbf{j})} J_{\mathrm{ij}} \sigma_{\mathbf{i}} \sigma_{\mathbf{j}}
$$

where $\sigma_{\mathrm{i}}, \sigma_{\mathrm{j}}= \pm 1$ are Ising spins on square lattice of size $L \times L=N$ and $J_{\mathrm{ij}}$ the random interaction between nearest neighbour spins, with the probability $x$ for $J_{i j}=-J$ and $1-x$ for 
$J_{i j}=+J$. The construction of ground states for a given sample and interactions is a complicated problem which cannot be solved by a Monte Carlo method [2]. It turns out that the relaxation process traps the system into low energy metastable states of very long lifetime [3]. Only special procedures such as the matching method (by hand [4] or computational [5]) or exact calculation [6] of partition function can give information at low or zero temperature. Here a new algorithm is proposed which is not only able to generate one ground state for each sample but some invariant properties for all the ground states.

The starting point is the exact correspondence between the frustration model and the Chinese postman's problem [7, 8], which is defined in the following way : a postman delivers mail along a set (graph G) of streets (edges) connecting cross-roads (nodes). What is the minimum round from the post office ? For even nodes only the solution is simply given by a Eulerian path but when odd nodes are present the postman walk is constrainted by repeated crossings between odd nodes.

Let us now recall the basic rules [9] of the frustration problem : the frustrated plaquettes are defined by an odd number of negative bonds on the perimeter. They are sources or sinks for a set of violated bonds called strings : a ground state energy is obtained when the total length of strings is minimum. It can be shown [10] that both rule sets are equivalent according to the dictionary :

$$
\begin{aligned}
& \text { odd nodes } \leftrightarrow \text { frustrated plaquettes } \\
& \text { repeated streets } \leftrightarrow \text { violated bonds } \\
& \text { round length } \leftrightarrow \text { frustration energy . }
\end{aligned}
$$

The polynomial algorithm of Edmonds which solves the Chinese postman's problem has been used here for generating ground states of frustration model. It is described in details elsewhere [10] and only summarized here :

(i) A primal problem is defined where a bond variable $x_{\mathrm{ij}}$ has value 0 or 1 for normal or violated bonds. Odd subsets $\mathrm{S}$ of frustrated plaquettes are delimited by frustrated contours. The integrality condition for $x_{\mathrm{ij}}$ is relaxed by adding linear constraints on these contours.

(ii) The dual problem associated to (i) is stated in terms of dual variables $y_{\mathbf{s}}$ related to frustrated contours. By analogy with dislocation theory $y_{\mathbf{s}}$ may be understood in terms of " line tension " of a frustrated contour analogous to "Volterra " circuit surrounding dislocation cores (frustrated plaquettes).

(iii) Using the weak theorem of complementary slackness [11] a simple criterion for optimality of primal and dual solutions is settled.

(iv) The spin configuration is progressively improved by flipping spin clusters having any irregular form. In contrast to Monte Carlo technique it is a non local algorithm which can overcome metastability.

(v) Two types of boundary conditions can be chosen by the algorithm for optimality : periodic or antiperiodic where the corresponding spins of opposite sides are parallel or antiparallel. This additional degree of freedom plays a crucial role in the description of the magnetic properties of ground states.

The rigidity of bonds is studied by a post-optimal algorithm. A rigid bond is by definition [10, 13] a violated bond (or non violated) for all the ground states.

A simple procedure is used to obtain the map of rigid bonds : starting from a ground state a violated bond is rigid if, for any $\varepsilon>0$ added to its weight $\left|J_{i j}\right|$ the optimality of the solution is not destroyed (similar procedure is possible for unviolated bonds). Hence, despite the exponential degeneracy for ground states [6], this algorithm has only $0\left(N^{2}\right)$ complexity [12] where $N$ denotes the total number of spins.

All the details of the numerical experimentation are reported in reference [10]. Let us just recall a few indications : the size is ranging from $10 \times 10$ up to $20 \times 20$, the concentration $x$ between 0 
and 0.5 and the number of samples : 248. Two types of defects have been found from the analysis of the morphology of these ground states. The fracture lines or contours of zero energy running from one side to another which proliferate at $x \gtrsim 0.15$. These defects, which have been conjectured previously $[5,14]$ appear as alternating cycles of an equal number of violated and non violated bonds in such a way that the energy is invariant under a change of the matching. The fracture line creeps along the corridor between the clusters of rigid bonds. It indicates a destruction of the macroscopic rigidity and implies that the magnetic long-range correlation cannot persist above $x \gtrsim 0.15$. Actually, in this region only small finite rigid clusters persist and the percolating rigid cluster is destroyed by fracture lines. In conclusion, here the ground states are disordered and the phase looks like superparamagnetism.

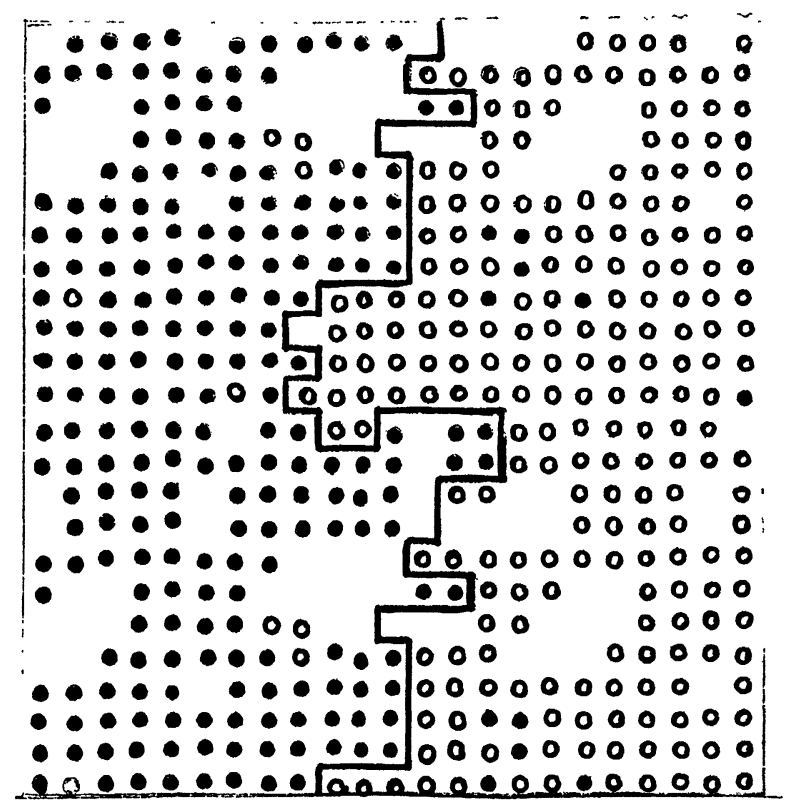

Fig. 1. - Random Antiphase State. The black and white circles represent the Ising up and down spins in a rigid configuration (same orientation for all the ground states). The loose spins the orientation of which change from one ground state to another are not marked. The magnetic wall separates two domains of up and down spins. Periodic boundary conditions are applied on the vertical edges while antiperiodic conditions work on the horizontal edges.

The magnetic walls appear when the samples are rigid, therefore below $x=0.15$, and are revealed by the fact that our algorithm chooses in almost all cases the antiperiodic boundary conditions. The frequency of occurrence of each type of defects increases abruptly at different values of $x$ : around $x \simeq 0.10$ for the magnetic walls and $x \simeq 0.15$ for the fracture lines. A magnetic wall is illustrated in figure 1 : it crosses the rigid bonds as well as living bonds. It is constituted by an unequal number of non violated negative bonds and violated positive bonds, in such a way that any alternation of the matching results in an increase of the energy. Since this defect exists in the range of rigidity, the magnetic correlation of the spins in the rigid cluster is equal to one although the total magnetization vanishes. It is therefore a highly correlated state, named Random Antiphase State, where the spin correlation function of the type $\langle S(0) S(R)\rangle_{0}^{2}$ (averaged over all the ground states) has its maximum value inside the percolating cluster of rigid bonds. 
2. Real space renormalization approach. - The renormalization in real space is a very simple method for the determination of a phase diagram by identifying the nature of the phase for each value of $T$ and $x$. Consider two spins connected through a cluster of spins with $\left\{J_{\mathrm{ij}}\right\}$ or $\left\{t_{\mathrm{ij}}=\tanh \left(J_{\mathrm{ij}} / T\right)\right\}$ a given configuration of the interactions. By summing over the spin states of the cluster (partial trace) an effective interaction is built $t^{\prime}\left(\left\{t_{\mathrm{ij}}\right\}\right)$ between the two spins. The iteration relation leads to a fixed value which identifies the nature of the phase, for instance $t^{\prime}=0$ for the paramagnetic phase or $t^{\prime}=1$ for the ferromagnetic state. For disordered systems the iteration procedure is applied on the probability distribution $p(t)$ rather than $t$. The integral equation for the "fixed " probability density is very complicated and no analytic solutions can be found. For square lattice a class of special clusters having the self dual symmetry turned out to be very efficient for the problem of conduction in random resistors [15] as well as dilution disorder problem [16]. The algebraic duality for the $t$ 's (Kramers-Wannier)

$$
t \rightarrow t_{\mathrm{D}}=\frac{1-t}{1+t}
$$

has a geometrical correspondence by the rule of construction of dual bonds intersecting the primal interactions. The self-dual clusters are the special class of clusters of spins which reproduce themselves by this simple rule. Beyond the trivial cluster of one bond, one finds the $\mathrm{H}$-cluster or Wheatstone bridge in two dimensions $(b=2)$ which possesses this property. The next cluster $(b=3)$ in 2 dimensions contains 13 bonds.

Let $t_{\mathrm{c}}=\tanh \frac{J}{T_{\mathrm{c}}}$ be the critical value corresponding to the critical temperature $T_{\mathrm{c}}$ for $x=0$. For each starting value $t$ the iteration relation leads to the fixed point of the paramagnetic phase $t=0$ for instance while the dual relation leads to the other fixed point, the ferromagnetic phase in this example.

The critical $t_{\mathrm{c}}$ separates the two regimes : the self-duality character of the cluster ensures that $t_{\mathrm{c}}$ is the exact value $: t_{\mathrm{c}}=\sqrt{2}-1$ for all $b$.

The present attempt deals with the spin glass model where negative interactions induce local frustration which plays a crucial role in the problem (previous attempts [17] based on square clusters concluded that no spin glass phase exists in 2 dimensions, but fixed points will be founded in this work from the H-cluster). The negative interactions, or negative $t$, destroy the standard selfduality relation since $t_{\mathrm{D}}$ becomes larger than 1 , i.e. $T$ complex. No symmetry around $x=0.5$ is expected in the phase diagram and the antiferromagnetic state will not be reached when $x \rightarrow 1$. However the expansion [18] of the critical temperature $T_{c}(x)$ as a function of $x$ for low value of $x$ fits remarkably well the $x$-expansion (see later). The self-duality symmetry is therefore preserved at least for small value of $x$.

The H-cluster gives the following iteration relation [16] :

$$
t^{\prime}=\frac{t_{1} t_{2}+t_{3} t_{4}+t_{5}\left(t_{1} t_{4}+t_{2} t_{3}\right)}{1+t_{1} t_{2} t_{3} t_{4}+t_{5}\left(t_{1} t_{3}+t_{2} t_{4}\right)}
$$

3. Numerical solution. - Let $P_{n}(t)$ the distribution function of the $t$ 's at the $n$th step of the iteration. We represent $P_{n}(t)$ by a table $T_{n}(i), 1<i<N$ where $N$ is the dimension of the table (practically $N=10^{3}$ ). Initially the table $T_{n}(i)$ is constructed from the values $-t$ and $+t$ with the respective concentration $x$ and $1-x$. A new table of $t^{\prime}$ is obtained by introducing five random values of $t_{i}$ in relation (2) leading to a new value $t^{\prime}$. At each step all the moments can be calculated and particularly the first one $M_{1}$ and the standard deviation. 
Three distinct fixed points have been identified in terms of $M_{1}$ and $\sigma$; the corresponding distribution laws are :

1. Ferromagnetism $M_{1}=1, \sigma=0, p(t)=\delta(t+1)$.

2. Paramagnetism $M_{1}=0, \sigma=0, p(t)=\delta(t)$.

3. Random antiphase state $M_{1}=0, \sigma=1, p(t)=\frac{1}{2}(\delta(t+1)+\delta(t-1))$.

The last point was called previously [17] the spin glass fixed point. Close to the RAS fixed point we have met a difficulty originating from the simultaneous cancellation of the denominator and the numerator. This intricated situation of indetermination calls for a careful analysis in the asymptotic approach of this fixed point. The nullity of the denominator occurs in the following conditions :

- frustration is present in the cluster;

- three values at least of the $t_{i}$ 's are equal to one.

The study of all the possible cases is straightforward.

In some few cases the indetermination can be removed. For other cases, it turns out that $t^{\prime}$ depends on the path in the appropriate space when the $t_{i}$ 's are going to 1 . But the major difficulty comes here from the nature of the iterated quantity - not a variable but a function. The sampling method for building the probability distribution by random choice of the $t_{i}$ is a discontinuous process for which no fixed values of $t^{\prime}$ are obtained by continuity. Then there is no means to remove the indetermination values in this asymptotic regime and we adopt the rule of rejecting all the occurrence of nullity in the denominator. With this rule, the convergence towards the RAS fixed points is very fast (between 10 and 20 steps). In the absence of any mathematical proof, we can only put forward a strong presumption of the stability of this fixed point based on the fact that when the numerical precision is doubled this fixed point remains stable. The separation lines in the phase diagram $T_{\mathrm{c}} / T_{\mathrm{c}}(0)$ versus $x$ is represented in figure 2 . The linear variation of $T_{\mathrm{c}}(x)$ for small $x$ corresponds to the first term of the linear expansion [18]

$$
T_{\mathrm{c}}(x) / T_{\mathrm{c}}(0)=1-3.2 x+0\left(x^{2}\right)
$$

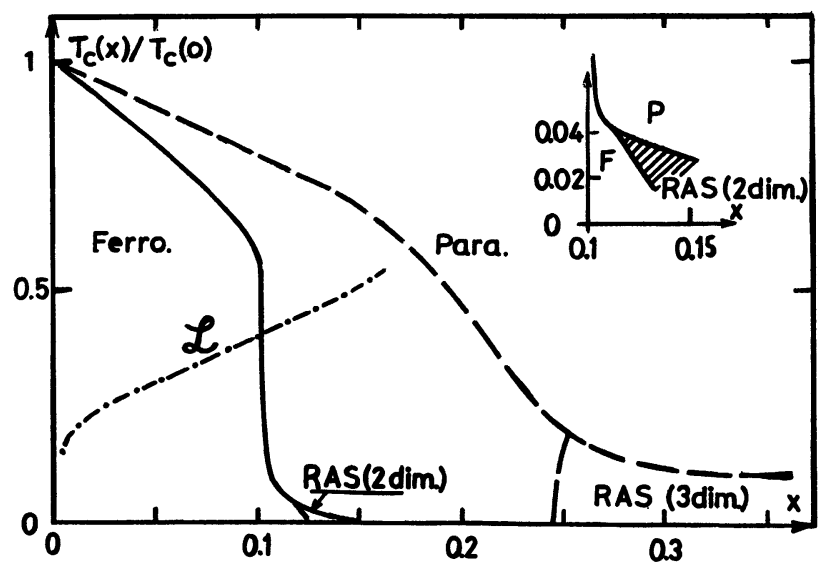

Fig. 2. - Phase diagram $T_{\mathrm{c}}(x)$. The full lines represent the 2 dimensional model of random $\pm J$ interactions. The separation line between the ferromagnetism and paramagnetism drops abruptly at $x=0.10$. Between $0.10 \leqslant x \leqslant 0.15$ and below $T_{c} / T_{c}(0) \leqslant 0.04$ a small domain is found where the fixed points are of the type Random Antiphase State (see insert). At three dimensions the domain of RAS is bigger and delimited by a dashed line. The dotted-dashed line $\mathcal{L}$ from the relation $\tanh (J / T)=1-2 x$ indicates states where the specific heat is finite and the internal energy has no singularity. 
by assuming that the nature of the phases is unchanged by varying $x$ (same class of universality). The agreement for the low value of $x$ is remarkable. At $x=0.1$ an abrupt decrease of the line separation at very small value of the reduced temperature $\simeq 0.04$. In the range of

$$
0.125 \leqslant x \leqslant 0.18 \text { and } 0.02 \leqslant T_{\mathrm{c}} / T_{\mathrm{c}}(0) \leqslant 0.04
$$

fixed points of RAS type are found as it is represented in the inset of the figure. It must be noticed that this pocket is located at very low temperature (less than 0.04 ) while the numerical saturation of the hyperbolic tangent $(t= \pm 1$ for $T \leqslant 0.04$ for a double numerical precision) assimilates the very low temperature $T=0.02$ at $T=0$.

In order to explore the generality of the previous phase diagram the same iteration has been performed with a Gaussian distribution function $p\left(J_{\mathrm{ij}}\right)$ of mean

$$
J_{0}=\left\langle J_{i j}\right\rangle=\tilde{J_{0}} / z \text { and } J=\left(\left\langle J_{i j}^{2}\right\rangle-\left\langle J_{i j}\right\rangle^{2}\right)^{1 / 2}=\tilde{J} / \sqrt{z}
$$

where $z$ is the coordination number of the lattice (here $z=4$ ). For $J=1$, the phase diagram has been obtained as a function of the reduced variable $T / \tilde{J}$ and $\tilde{J_{0}} / \tilde{J_{\text {. }}}$. For $\tilde{J_{0}} / \tilde{J} \gtrsim 1.02$ only paramagnetic and ferromagnetic fixed points are obtained. Below $\tilde{J_{0}} / \tilde{J} \lesssim 0.8$ we have found only the paramagnetic fixed point even for the very low temperature $T / \tilde{J} \simeq 0.05$. The line of phase separation drops down abruptly at $\tilde{J_{0}} / \tilde{J} \lesssim 1.02$ and in this small interval $0.8 \lesssim \tilde{J_{0}} / \tilde{J} \lesssim 1.02$, fixed RAS are obtained below $T_{\mathrm{c}} / \tilde{J} \lesssim 0.1$. There is a great similarity between the Gaussian random $p(J)$ and the $\pm J$ in the occurrence of the three phases : paramagnetic, ferromagnetic and RAS. [It is remarkable that the critical value for the occurrence of ferromagnetism at low temperature near $x=0.1$ (see Fig. 2) corresponds to the value $\widetilde{J_{0}} / \tilde{J}=1.02$ where the tail of the Gaussian law centred at this value corresponds to $10 \%$ of negative interactions among all the bonds.] With the next cluster $b=3$ the same result is obtained qualitatively.

The previous results have been extended in $d=3$ by using the octahedral cluster where a plaquette replaces now the intermediate branch of a Wheatstone bridge. Contrasting with the $d=2$ case and for $x=0$ in the pure ferromagnetic case we have found $T_{c}(0) / J=5.43$ instead of 4.510 the accepted value of the 3 dimensional Ising model. For the $\pm J$ distribution the phase diagram is shown in figure 2 (dotted line). The domain of the RAS is extended for $0.25 \leqslant x \leqslant 0.5$ and in temperature, up to $T_{\mathrm{c}}(x) / J=1$. The three separation lines are the following :

- Ferromagnetism-paramagnetism where

$$
T_{\mathrm{c}}(x) / T_{\mathrm{c}}(0)=1-2.176 x+0\left(x^{2}\right) .
$$

This result confirms the expansion [18] in $x$ from the high temperature [19] expansion of the susceptibility.

- RAS-paramagnetism where the line is almost horizontal at $T_{\mathrm{c}}(x) / J \simeq 1$.

- RAS-ferromagnetism line almost vertical at $x=0.24$.

These three lines intersect at a point where the high temperature series of the susceptibility departs from the power law divergence. Actually at $x \geqslant 0.25$, the analysis of the expansion in terms of Padé approximants reveals an instability of the pole at $T_{\mathrm{c}}$ (from $x \lesssim 0.25$ ) and gives a pair of complex conjugated poles. This diagram is in agreement with the previous scheme of decimation found by different authors [17].

4. Discussions and conclusions. - There are very few exact results in the problem of spin glasses. We compare our results with two known exact results in $d=2$.

4.1 DisAPPEARANCE OF FERROMAGNETISM. - From the gauge invariance symmetry it has been shown [20] that a regular line $\mathcal{L}$ in the $(T, x)$ plane given by the equation

$$
t=\tanh (J / T)=1-2 x
$$


plays a very special role. For instance on $\mathcal{L}$ the specific heat is always finite $(\alpha<0)$. Let $x_{0}$ the the concentration where this line $\mathcal{L}$ intersects the separation line between the ferromagnetism and the paramagnetism (see Fig. 2). It can be shown [20] that the magnetization vanishes at any temperature for $x_{0}<x<0.5$. Therefore this value indicates the disappearance of ferromagnetism while the derivative $\mathrm{d} T_{\mathrm{c}}(x) / \mathrm{d} x=\infty$ at $x_{0}$ showing that $T_{\mathrm{c}}(x)$ is a non-differentiable function at $x_{0}$. In figure $2, \mathcal{L}$ is represented and $x_{0}=0.105, T_{\mathrm{c}}\left(x_{0}\right)=0.95 \mathrm{~J}$. Note that the ferromagnetic phase extends lightly beyond $x_{0}$.

4.2 RigIDITY AND RANDOM ANTIPHASE STATE. - The zero-value of the spontaneous magnetization indicates the absence of ferromagnetic order but does not exclude the possibility of an ordered phase of different nature. The extension [21] of the Peierls argument to the present model of frustration is the following : when the ground states admit fracture lines or contours of zero energy of finite length - " rigid " ground states - then a finite critical temperature exists which separates the paramagnetic phase from a condensed phase. This condition is sufficient (but non necessary in general) and is fulfilled here in the intermediate range of concentration :

$$
0.10 \leqslant x \leqslant 0.15
$$

where only small and finite fracture lines have been observed. We believe that the disappearance of the ferromagnetic order above $x=0.10$ provides a new rigid phase, the RAS.

4.3 CONJECtURES ABOUt THE RANDOM ANTIPHASE STATE (RAS) AND CONClUSIONS. - The existence of this new RAS contradicts some results obtained by different authors [2, 17], but the critical temperature in this range is very small, less than $0.04 T_{\mathrm{c}}(0)$, and therefore the numerical studies are very difficult. Here, it is by using two different techniques that this new phase is observed for the first time at $d=2$. Numerical studies for samples of larger size $50 \times 50$ as well as exact calculation of partition functions are in progress in order to confirm these results. This RAS is characterized by erratic magnetic walls running in rigid ground states. This sort of morphology of the ground states indicates that the Ising spin variables $(n=1)$ which describe the ferromagnetic phase are not sufficient to describe a RAS. More complex variables $(n>1)$ are necessary in order to describe the structure. This situation recalls the Ising models of competing interactions [22] where the modulated phases need the introduction of new field variables where the dimensionality of the spins as well as the universality class are changed.

Acknowledgments. - The authors acknowledge F. Barahona and J. P. Uhry from the department of Applied Mathematics of the University of Grenoble, for their initiation to graph theory and the active cooperation on the application of the Edmond's algorithm to the frustration problem as well as the numerical simulation.

\section{References}

[1] Rammal, R. and Souletie, J., Spin Glasses, to be published in Magnetism of Metals and Alloys, Editor M. Cyrot (North-Holland) 1982.

[2] Binder, K., Kinzel, W., Proceedings of the International Conference on Disordered Systems and Localisation, Rome 1981, C. Castellani, C. Di Castro, L. Peliti eds (Springer Verlag) 1982.

[3] Rammal, R., Suchail, R. and Maynard, R., Solid State Commun. 32 (1979) 487.

[4] Vannimenus, J. and Toulouse, G., J. Phys. C : Solid State Phys. 10 (1977) L-537.

[5] Bieche, I., Maynard, R., Rammal, R. and Uhry, J. P., J. Phys. A : Math. Gen. 13 (1980) 2253.

[6] Morgenstern, I. and Binder, K., Phys. Rev. B 22 (1980) 288.

[7] Mei-Ko Kwan, Chinese Math. 1 (1962) 273. 
[8] Edmonds, J., Operation Research 13 Suppl. 1 (1965) 373.

EDMONDS, J. and Johnson, E. L., Math. Program. 5 (1973) 88.

[9] Toulouse, G., Commun. Phys. 2 (1977) 115.

[10] Barahona, F., Maynard, R., Rammal, R. and Uhry, J. P., J. Phys. A : Math. Gen. 15 (1982) 673.

[11] Orlova, G. I., Dorman, Y. G., Eng. Cybern. 10 (1972) 502.

[12] Barahona, F., to be published.

[13] Vannimenus, J., Maillard, J. P. and de Seze, L., J. Phys. C : Solid State Phys. 12 (1979) 4523.

[14] Bray, A. and Moore, M. A., J. Phys. F : Met. Phys. 7 (1977) L-333.

[15] Bernasconi, J., Phys. Rev. B 18 (1978) 2185.

[16] Levy, S. V. F., Tsallis, C., Curodo, E. M. F., Phys. Rev. B 21 (1980) 2991 ;

Tsallis, C., LeVY, S. V. F., J. Phys. C 13 (1980) 465.

[17] Southern, B. W., Young, A. P., J. Phys. C 10 (1977) 2179.

[18] Rammal, R., thèse, Université de Grenoble (1981).

[19] Matsubara, F., Katsura, S., J. Phys. C 10 (1977) 4361.

[20] Noshimori, H., J. Phys. C 13 (1980) 4071.

[21] Süto, A., J. Stat. Phys. 23 (1980) 203.

[22] Krinsky, S., Mukamel, D., Phys. Rev. B 16 (1977) 2313. 